\title{
Spectral diagonal ensemble Kalman filters
}

\author{
I. Kasanický ${ }^{1}$, J. Mandel ${ }^{1,2}$, and M. Vejmelka ${ }^{1}$ \\ ${ }^{1}$ Institute of Computer Science, Academy of Sciences of the Czech Republic, Prague, Czech Republic \\ ${ }^{2}$ Department of Mathematical and Statistical Sciences, University of Colorado Denver, Denver, CO, USA
}

Correspondence to: J. Mandel (jan.mandel@gmail.com)

Received: 30 December 2014 - Published in Nonlin. Processes Geophys. Discuss.: 27 January 2015

Revised: 26 July 2015 - Accepted: 29 July 2015 - Published: 18 August 2015

\begin{abstract}
A new type of ensemble Kalman filter is developed, which is based on replacing the sample covariance in the analysis step by its diagonal in a spectral basis. It is proved that this technique improves the approximation of the covariance when the covariance itself is diagonal in the spectral basis, as is the case, e.g., for a second-order stationary random field and the Fourier basis. The method is extended by wavelets to the case when the state variables are random fields which are not spatially homogeneous. Efficient implementations by the fast Fourier transform (FFT) and discrete wavelet transform (DWT) are presented for several types of observations, including high-dimensional data given on a part of the domain, such as radar and satellite images. Computational experiments confirm that the method performs well on the Lorenz 96 problem and the shallow water equations with very small ensembles and over multiple analysis cycles.
\end{abstract}

\section{Introduction}

Data assimilation consists of incorporating new data periodically into computations in progress, which is of interest in many fields, including weather forecasting (e.g., Kalnay, 2003; Lahoz et al., 2010). One data assimilation method is filtering (e.g., Anderson and Moore, 1979), which is a sequential Bayesian estimation of the state at a given time given the data received up to that time. The probability distribution of the system state is advanced in time by a computational model, and modified using the Bayes theorem. In the methods considered here, data is assimilated in discrete time steps, called analysis cycles, and the probability distribution of the state is represented by an ensemble. The analysis is based on the state covariance, thus making a tacit assump- tion that the state probability distribution is at least close to Gaussian. When the state covariance is given externally, Bayesian estimation becomes the classical optimal statistical interpolation (OSI). The Kalman filter (KF) uses the same computation as OSI in the analysis, but it evolves the covariance matrix of the state in time along with the model state. Since the covariance matrix can be large, the KF is not suitable for high-dimensional systems. The ensemble Kalman filter (EnKF) (Evensen, 2009) replaces the state covariance by the sample covariance computed from an ensemble of simulations, which represent the state probability distribution. It can be proved that the EnKF converges to the KF in the large ensemble limit (Kwiatkowski and Mandel, 2015; Le Gland et al., 2011; Mandel et al., 2011) in the linear and Gaussian case, but an acceptable approximation may require hundreds of ensemble members (Evensen, 2009), because of spurious long-distance correlations in the sample covariance due to its low rank. Localization techniques (e.g., Anderson, 2001; Furrer and Bengtsson, 2007; Hunt et al., 2007) essentially suppress long-distance covariance terms (Sakov and Bertino, 2011), which improves EnKF performance for small ensembles.

The fast Fourier transform (FFT) EnKF (Mandel et al., 2010a, b) was proposed as an alternative approach to localization, based on replacing the sample covariance in the EnKF by its diagonal in the Fourier space. This approach is motivated by the fact that a random field in cartesian geometry is second-order stationary (i.e., the covariance between the values at two points depends only on their distance vector) if and only if its covariance in the Fourier space is diagonal (e.g., Pannekoucke et al., 2007). On a sphere, an isotropic random field has diagonal covariance in the basis of spherical harmonics (Boer, 1983), so similar algorithms can be developed there as well. However, the stationarity as- 
sumption does not allow the covariance to vary spatially. For this reason, the FFT EnKF was extended to wavelet EnKF (Beezley et al., 2011). The use of wavelets results in an automatic localization, which varies in space adaptively. For wavelets, the effect of the diagonal spectral model is equivalent to a weighted spatial averaging of local covariance functions (Pannekoucke et al., 2007). Diagonal matrices are inexpensive to manipulate computationally, but implementing the multivariate case and general observation functions is not straightforward.

Spectral diagonal covariance models and their estimation from an ensemble of realizations are not new. Diagonal spectral modeling and, more generally, sparse spectral covariance modeling, have been used for the background covariance in data assimilation in meteorology for some time. The optimal statistical interpolation system from Parrish and Derber (1992) was based on a diagonal covariance model in spherical harmonics, which were already used as horizontal basis functions in the numerical weather prediction code with a change of state variables into physically balanced analysis variables, and it has been used in operational weather forecasting for a long time. Estimates of background covariance from an ensemble, called flow-dependent covariance, in combinations with spectral covariance models have been used in variational data assimilation (e.g., Buehner, 2005; Buehner and Charron, 2007; Berre et al., 2007; Varella et al., 2011), leading to hybrid EnKF-3DVAR (3-D variational) methods. Another hybrid formulation in EnKF was proposed in Hamill and Snyder (2000, Eq. 4), who proposed a linear combination of sample covariance, different in every analysis cycle, and background spectral diagonal covariance from Parrish and Derber (1992), which does not change over analysis cycles. The ECMWF 3DVAR system (Courtier et al., 1998) also used diagonal covariance in spherical harmonics for the background covariance. A diagonal model in the Fourier space for homogeneous 2-D error fields, with physically balanced cross-covariances, was proposed in Berre (2000). The Fourier diagonalization approach was extended by Pannekoucke et al. (2007) to sparse representation of the background covariance by thresholding wavelet coefficients, and into a combined spatial and spectral localization by Buehner and Charron (2007). The balanced update and localization in the EnKF using the stream function-velocity potential representation were studied in Kepert (2009).

Further developments in the history of background covariance modeling in variational algorithms include construction of non-separable formulation (Courtier et al., 1998; Fisher and Andersson, 2001; Pannekoucke, 2009), representation of balances between variables in order to obtain a more realistic multivariate formulation (Derber and Bouttier, 1999; Fisher, 2003; Weaver et al., 2005), representation of heterogeneity using a physically/spectrally localized formulation (nonseparable wavelet formulation (Deckmyn and Berre, 2005; Fisher and Andersson, 2001), separable formulation based on diffusion operator (Weaver and Courtier, 2001) or recur- sive filters (Purser et al., 2003), and a non-separable formulation based on hybridization of diffusion and wavelets (Pannekoucke, 2009). Formulations such as the diffusion operator or the recursive filter are related to the diagonal assumption here, they involve covariance models with a relatively small number of parameters and thus free of sampling noise but estimated from an ensemble directly (Pannekoucke and Massart, 2008; Michel, 2013; Pannekoucke et al., 2014). Similar filtering strategies can be employed to improve the estimation and the design of covariance formulations using results on the estimation of variances and length scales (Berre et al., 2007; Raynaud et al., 2009; Raynaud and Pannekoucke, 2013; Ménétrier et al., 2015). The formulation of the background error covariance model using the diagonal assumption and a product of linear operator (such as the discrete Fourier or wavelet transform here) is widely used in variational literature to build covariance models in high dimension (e.g., Courtier et al., 1998; Fisher and Andersson, 2001; Weaver and Courtier, 2001).

The idea of using a covariance model to benefit sample noise reduction is known; however, as far as we know no reference has been published to document the real advantage of this method in improvements to the performance of the EnKF. The paper provides a preliminary test, within an academic setting, of the techniques of employing parametric covariance in the EnKF, while the existing literature is focused on the opposite direction, the use of ensembles to provide estimates for the variational framework, known as "hybrid formulation". Specifically, the use of spectral covariance modeling in each EnKF analysis cycle to reduce the ensemble size seems to be new. The main reason could be that it requires building a covariance matrix parameterization, which represents a real cost in terms of technology investment for numerical weather prediction codes.

While modeling background covariances typically uses multiple sources including historical data, the EnKF builds the covariance in every analysis cycle from the ensemble itself. In this paper, we prove that replacing the sample covariance by its spectral diagonal improves the approximation when the covariance itself is diagonal in the spectral space, as is the case, e.g., when the state is a second-order stationary random field and a Fourier basis is used. The result, however, is general and it applies to an arbitrary orthogonal basis, including wavelets. We also develop computationally efficient spectral EnKF algorithms, which take advantage of the diagonal form of the covariance, in the multivariate case and for several important classes of observations. We demonstrate the methods on computational examples with the Lorenz 96 system and shallow water equations, which show that good performance can be achieved with very small ensembles. 


\section{Notation}

Vectors in $\mathbb{R}^{n}$ or $\mathbb{C}^{n}$ are typeset as $\boldsymbol{u}$ and understood to be columns. Random vectors are typeset as $\boldsymbol{X}$. The entry $i$ of $\boldsymbol{X}$ is denoted by $(\boldsymbol{X})_{i}$ or $x_{i}$. Matrices (random or deterministic) are typeset as $\mathbf{A}$, and $\mathbf{A}^{*}$ is the transpose or conjugate transpose in the complex case. The entry $i, j$ of matrix $\mathbf{A}$ is denoted by $(\mathbf{A})_{i, j}$ or $a_{i, j}$, and $\mathbf{A}=\left[\boldsymbol{a}_{1}, \ldots, \boldsymbol{a}_{n}\right]$ is the writing of a matrix as a collection of columns. Nonlinear operators are typeset as $\mathcal{M}$. The mean value is denoted by $E[\cdot]$, and Var is the variance. $N(0,1)$ is the normal (Gaussian) distribution with zero mean and unit variance, and $N(\boldsymbol{m}, \mathbf{C})$ is the multivariate normal distribution with mean $\boldsymbol{m}$ and covariance C. The Euclidean norm of a vector is $|\boldsymbol{u}|=\left(\sum_{i=1}^{n}\left|u_{i}\right|^{2}\right)^{1 / 2}$. The Frobenius norm of a matrix, also known as Hilbert-Schmidt norm, is $|\mathbf{A}|_{\mathrm{F}}=\left(\sum_{i=1}^{m} \sum_{j=1}^{n}\left|a_{i, j}\right|^{2}\right)^{1 / 2}$.

\section{Kalman filter and ensemble Kalman filter}

The state of the system at time $t$ is described by a random vector $\boldsymbol{X}_{t}$ of length $n$. The system evolution between two times $t_{1}$ and $t_{2}$ is given by a function $\mathcal{M}\left(\cdot, t_{1}, t_{2}\right)$, so that

$\boldsymbol{X}_{t_{2}}^{\mathrm{f}}=\mathcal{M}\left(\boldsymbol{X}_{t_{1}}^{\mathrm{a}}, t_{1}, t_{2}\right)$.

The goal of the KF (Kalman, 1960) is to correct the forecast state of the system $\boldsymbol{X}_{t}^{\mathrm{f}}$ to obtain the analysis estimate $\boldsymbol{X}_{t}^{\mathrm{a}}$ of the true state $\boldsymbol{X}_{t}$, given noisy observations $\boldsymbol{Y}_{t}=\mathbf{H}_{t} \boldsymbol{X}_{t}+\boldsymbol{\epsilon}_{t}$, where $\mathbf{H}_{t}$ is an observation operator, i.e., a mapping from state space to a data space, and $\boldsymbol{\epsilon}_{t} \sim N\left(\mathbf{0}, \mathbf{R}_{t}\right)$. When the distributions of the state $\boldsymbol{X}_{t}$ and the data error are Gaussian, the analysis satisfies

$\boldsymbol{X}_{t}^{\mathrm{a}}=\boldsymbol{X}_{t}^{\mathrm{f}}-\mathbf{K}_{t}\left(\mathbf{H}_{t} \boldsymbol{X}_{t}^{\mathrm{f}}-\boldsymbol{Y}_{t}\right)$,

$\mathbf{K}_{t}=\mathbf{C}_{t} \mathbf{H}_{t}^{*}\left(\mathbf{H}_{t} \mathbf{C}_{t} \mathbf{H}_{t}^{*}+\mathbf{R}_{t}\right)^{-1}$,

where $\mathbf{C}_{t}$ is the covariance of the forecast $\boldsymbol{X}_{t}^{\mathrm{f}}$, and $\mathbf{K}_{t}$ is called the Kalman gain. In the KF, the state is represented by its mean and covariance, and the mean is transformed also by Eqs. (1) and (2). In the rest of the paper, we will drop the time index $t$ and the superscript $\mathrm{f}$, unless there is a danger of confusion.

In the EnKF, the analysis formulas (Eqs. 1,2) are applied to each ensemble member, with the covariance replaced by the sample covariance from the ensemble. The resulting ensemble, however, would underestimate the analysis covariance, which is corrected by a data perturbation by sampling from the data error distribution (Burgers et al., 1998). Denote by $\boldsymbol{X}^{1}, \ldots, \boldsymbol{X}^{N}$ the forecast ensemble, created either by a perturbation of a background state or by evolving each analysis ensemble member from the previous time step independently by Eq. (1). Then, the analysis ensemble members are
$\boldsymbol{X}^{\mathrm{a}, j}=\boldsymbol{X}^{j}-\mathbf{C}^{N} \mathbf{H}^{*}\left(\mathbf{H} \mathbf{C}^{N} \mathbf{H}^{*}+\mathbf{R}\right)^{-1}\left(\mathbf{H} \boldsymbol{X}^{j}-\boldsymbol{Y}^{j}\right)$,

where the sample covariance matrix is

$$
\begin{aligned}
& \mathbf{C}^{N}=\frac{1}{N-1} \sum_{j=1}^{N}\left(\boldsymbol{X}^{j}-\overline{\boldsymbol{X}}\right)\left(\boldsymbol{X}^{j}-\overline{\boldsymbol{X}}\right)^{*}, \\
& \overline{\boldsymbol{X}}=\frac{1}{N} \sum_{j=1}^{N} \boldsymbol{X}^{j},
\end{aligned}
$$

and $\boldsymbol{Y}^{j}=\boldsymbol{Y}+\boldsymbol{\tau}^{j}$ are the perturbed observations, with $\boldsymbol{\tau}^{j} \sim N(\mathbf{0}, \mathbf{R})$ independent.

The advantage of the EnKF update (Eqs. 3, 4) is that it can be implemented efficiently without forming the sample covariance matrix $\mathbf{C}^{N}$ explicitly (e.g., Mandel et al., 2009, Eq. 15). On the other hand, the rank of the matrix $\mathbf{C}^{N}$ is at most $N-1$, while the number of significant modes can be higher. In the usual case, when $N$ is small, the low rank of the approximation $\mathbf{C}^{N}$ of the true forecast covariance $\mathbf{C}$ causes spurious long-range correlations, which are the biggest drawback of the EnKF.

\section{Spectral diagonal EnKF}

Let $\mathbf{F}$ be an orthonormal transformation matrix, which transforms each ensemble member to spectral space, and denote each transformed ensemble member by the additional subscript $\mathrm{F}, \boldsymbol{X}_{\mathrm{F}}^{j}=\mathbf{F} \boldsymbol{X}^{j}, j=1, \ldots, N$. Since the transformation is orthonormal, the inverse transformation is $\mathbf{F}^{*}$, so $\mathbf{F}^{*} \boldsymbol{X}_{\mathrm{F}}^{j}=\boldsymbol{X}^{j}$ for each $j=1, \ldots, N$. The columns of the inverse transform matrix $\mathbf{F}^{*}$ are the spectral basis elements $\boldsymbol{u}_{1}, \ldots, \boldsymbol{u}_{n}$, i.e., $\mathbf{F}=\left[\boldsymbol{u}_{1}, \ldots, \boldsymbol{u}_{n}\right]^{*}$. We will also denote the sample covariance of the transformed ensemble with the additional subscript $\mathrm{F}$,

$$
\begin{aligned}
& \mathbf{C}_{\mathrm{F}}^{N}=\frac{1}{N-1} \sum_{j=1}^{N}\left(\boldsymbol{X}_{\mathrm{F}}^{j}-\overline{\boldsymbol{X}}_{\mathrm{F}}\right)\left(\boldsymbol{X}_{\mathrm{F}}^{j}-\overline{\boldsymbol{X}}_{\mathrm{F}}\right)^{*}=\mathbf{F C}^{N} \mathbf{F}^{*}, \\
& \overline{\boldsymbol{X}}_{\mathrm{F}}=\frac{1}{N} \sum_{j=1}^{N} \boldsymbol{X}_{\mathrm{F}}^{j} .
\end{aligned}
$$

The idea of the spectral diagonal Kalman filter is to replace the sample covariance in the analysis formula (Eq. 3) by only the diagonal elements of sample covariance in spectral space:

$$
\begin{aligned}
& \mathbf{D}_{\mathrm{F}}^{N}=\mathbf{C}_{\mathrm{F}}^{N} \circ \mathbf{I}=\left[\begin{array}{llll}
c_{1,1} & 0 & \cdots & 0 \\
0 & c_{2,2} & & \vdots \\
\vdots & & \ddots & 0 \\
0 & \cdots & 0 & c_{n, n}
\end{array}\right], \\
& c_{i, i}=\frac{1}{N-1} \sum_{j=1}^{N}\left|\left(\boldsymbol{X}_{\mathrm{F}}^{j}\right)_{i}-\left(\overline{\boldsymbol{X}}_{\mathrm{F}}\right)_{i}\right|^{2},
\end{aligned}
$$

where o stands for the Schur product, i.e., element-wise multiplication. The entries $c_{i, i}$ are the sample variances, com- 
puted without forming the whole matrix $\mathbf{C}_{\mathrm{F}}^{N}$. The diagonal model is transformed back to physical space as

$\mathbf{D}^{N}=\mathbf{F}^{*} \mathbf{D}_{\mathrm{F}}^{N} \mathbf{F}$,

and the proposed analysis update is then

$$
\boldsymbol{X}^{\mathrm{a}, j}=\boldsymbol{X}^{j}-\mathbf{D}^{N} \mathbf{H}\left(\mathbf{H D}^{N} \mathbf{H}^{*}+\mathbf{R}\right)^{-1}\left(\mathbf{H} \boldsymbol{X}^{j}-\boldsymbol{Y}^{j}\right) .
$$

\section{Error analysis}

We will now compare the expected errors of the sample covariance and its spectral diagonal model (Eq. 7). The analysis extends results for a sample covariance formula with known zero mean (Furrer and Bengtsson, 2007; Mallat, 1998) by taking into account the sample mean in Eq. (4). This extension is important because the mean of the ensemble members is not known in practice and an estimate must be used instead.

Assume that the ensemble members $\boldsymbol{X}^{i} \sim N(\boldsymbol{\mu}, \mathbf{C})$ are i.i.d. (independent and identically distributed). (In the EnKF, the ensemble members after the first analysis cycle are not independent, because the sample covariance in the analysis step ties them together, but they converge to independent random vectors as the ensemble size $N \rightarrow \infty$ (Le Gland et al., 2011; Mandel et al., 2011).)

Using Lemma 1 from the Appendix and the fact that the Frobenius norm is invariant to orthogonal transformations, we have in any case,

$$
\begin{aligned}
E\left[\left|\mathbf{C}-\mathbf{C}^{N}\right|_{\mathrm{F}}^{2}\right] & =E\left[\left|\mathbf{C}_{\mathrm{F}}-\mathbf{C}_{\mathrm{F}}^{N}\right|_{\mathrm{F}}^{2}\right] \\
& =\frac{1}{N-1} \sum_{i, j=1}^{n}\left(\left|\left(\mathbf{C}_{\mathrm{F}}\right)_{i, j}\right|^{2}+\left(\mathbf{C}_{\mathrm{F}}\right)_{i, i}\left(\mathbf{C}_{\mathrm{F}}\right)_{j, j}\right) \\
& =\frac{2}{N-1} \sum_{i, j=1}^{n}\left|\left(\mathbf{C}_{\mathrm{F}}\right)_{i, j}\right|^{2} \\
& +\frac{1}{N-1} \sum_{\substack{i, j=1 \\
i \neq j}}^{n}\left(\mathbf{C}_{\mathrm{F}}\right)_{i, i}\left(\mathbf{C}_{\mathrm{F}}\right)_{j, j}
\end{aligned}
$$

The purpose of the spectral transformation is to bring the covariance to a diagonal form $\mathbf{C}_{\mathrm{F}}=\mathbf{F C F} \mathbf{F}^{*}$, where $\mathbf{F}$ is orthogonal transformation. Specifically, the rows of the spectral transformation matrix $\mathbf{F}^{*}$ are orthonormal eigenvectors of the covariance $\mathbf{C}$. This is the situation, e.g., when the ensemble members $\boldsymbol{X}^{i}$ are sampled from a second-order stationary random field on a rectangular mesh and the Fourier basis is used. Then, using $\left(\mathbf{C}_{\mathrm{F}}\right)_{i, j}=0$ for $i \neq j$, we get that the expected error of the spectral diagonal model consists of the diagonal terms in the frequency domain only:

$$
\begin{aligned}
E\left[\left|\mathbf{C}-\mathbf{D}^{N}\right|_{\mathrm{F}}^{2}\right] & =E\left[\left|\mathbf{C}_{\mathrm{F}}-\mathbf{C}_{\mathrm{F}}^{N} \circ \mathbf{I}\right|_{\mathrm{F}}^{2}\right] \\
& =\frac{1}{N-1} \sum_{i=1}^{n}\left(\left|\left(\mathbf{C}_{\mathrm{F}}\right)_{i, i}\right|^{2}+\left(\mathbf{C}_{\mathrm{F}}\right)_{i, i}\left(\mathbf{C}_{\mathrm{F}}\right)_{i, i}\right) \\
& =\frac{2}{N-1} \sum_{i=1}^{n}\left|\left(\mathbf{C}_{\mathrm{F}}\right)_{i, i}\right|^{2}
\end{aligned}
$$

Consequently,

$E\left[\left|\mathbf{C}-\mathbf{D}^{N}\right|_{\mathrm{F}}^{2}\right] \leq E\left[\left|\mathbf{C}-\mathbf{C}^{N}\right|_{\mathrm{F}}^{2}\right]$

with equality only if $\left(\mathbf{C}_{\mathrm{F}}\right)_{i, i}\left(\mathbf{C}_{\mathrm{F}}\right)_{j, j}=0$, for all $i \neq j$, i.e., only in the degenerate case when the covariance $\mathbf{C}_{\mathrm{F}}$ and thus $\mathbf{C}$ have a rank of at most one.

To assess the improvement gained by the spectral diagonal model in Eq. (11), denote the eigenvalues of $\mathbf{C}$ by $\lambda_{i}=\left(\mathbf{C}_{\mathrm{F}}\right)_{i, i}$, and without loss of generality assume that $0 \leq \lambda_{1} \leq \lambda_{2} \leq \cdots \leq \lambda_{n}$. The error estimates (Eqs. 9, 10) can be now written as

$$
E\left[\left|\mathbf{C}-\mathbf{C}^{N}\right|_{\mathrm{F}}^{2}\right]=\frac{2}{N-1} \sum_{i=1}^{n} \lambda_{i}^{2}+\frac{1}{N-1} \sum_{\substack{i, j=1 \\ i \neq j}}^{n} \lambda_{i} \lambda_{j},
$$

and

$$
E\left[\left|\mathbf{C}-\mathbf{D}^{N}\right|_{\mathrm{F}}^{2}\right]=\frac{2}{N-1} \sum_{i=1}^{n} \lambda_{i}^{2} .
$$

Note that

$$
\begin{aligned}
\left(\sum_{i=1}^{n} \lambda_{i}\right)^{2} & =\sum_{i, j=1}^{n} \lambda_{i} \lambda_{j}=\sum_{i, j=1, i \neq j}^{n} \lambda_{i} \lambda_{j} \\
& +\sum_{i=1}^{n} \lambda_{i}^{2} \geq \sum_{i=1}^{n} \lambda_{i}^{2},
\end{aligned}
$$

which shows that the error of the sample covariance depends on the $\ell^{1}$ norm of the eigenvalues sequence,

$$
\begin{aligned}
E\left[\left|\mathbf{C}-\mathbf{C}^{N}\right|_{\mathrm{F}}^{2}\right] & =\frac{1}{N-1}\left(\sum_{k=1}^{n} \lambda_{k}^{2}+\left(\sum_{k=1}^{n} \lambda_{k}\right)^{2}\right) \\
& =\frac{1}{N-1}\left(\left|\left\{\lambda_{k}\right\}_{k=1}^{n}\right|_{\ell^{2}}^{2}+\left|\left\{\lambda_{k}\right\}_{k=1}^{n}\right|_{\ell^{1}}^{2}\right),
\end{aligned}
$$

while the error of the spectral diagonal model depends only on the $\ell^{2}$ norm,

$E\left[\left|\mathbf{C}-\mathbf{D}^{N}\right|_{\mathrm{F}}^{2}\right]=\frac{2}{N-1}\left|\left\{\lambda_{k}\right\}_{k=1}^{n}\right|_{\ell^{2}}^{2}$,

which is weaker than the $\ell^{1}$ norm as the state space dimension $n \rightarrow \infty$. The improvement depends on the rate of decay of the eigenvalues as the index $k \rightarrow \infty$. Note that the 
eigenvalues of the covariance (if it exists) of a random element in an infinitely dimensional Hilbert space must satisfy the trace condition $\sum_{k=1}^{\infty} \lambda_{k}<\infty$ (e.g., Da Prato, 2006). The eigenvalues of the covariance in many physical systems obey a power law, $\lambda_{k} \approx k^{-\alpha}$ with $\alpha>1$ (e.g., Gaspari and Cohn, 1999). Suppose that $\lambda_{k}=c k^{-\alpha}$ and $n \rightarrow \infty$. Then,

$$
\begin{aligned}
& \left|\left\{\lambda_{k}\right\}_{k=1}^{n}\right|_{\ell^{2}}^{2} \rightarrow \sum_{k=1}^{\infty} k^{-2 \alpha} \approx \int_{1}^{\infty} x^{-2 \alpha} \mathrm{d} x=\frac{1}{2 \alpha-1}, \\
& \left|\left\{\lambda_{k}\right\}_{k=1}^{n}\right|_{\ell^{1}}^{2} \rightarrow \sum_{k=1}^{\infty} k^{-\alpha} \approx \int_{1}^{\infty} x^{-\alpha} \mathrm{d} x=\frac{1}{\alpha-1},
\end{aligned}
$$

which gives the error ratio $E\left[\left|\mathbf{C}-\mathbf{D}^{N}\right|_{\mathrm{F}}^{2}\right] / E\left[\left|\mathbf{C}-\mathbf{C}^{N}\right|_{\mathrm{F}}^{2}\right]$ $\rightarrow 0$ as $\alpha \rightarrow 1_{+}$, i.e., when the eigenvalues decay slowly. Other considerations of similar ratios can be found in Furrer and Bengtsson (2007).

Several concluding remarks are in order. Furrer and Bengtsson (2007) consider tapering to the diagonal in the physical space, but diagonal covariance in the physical space is never used in applications. The present method is EnKF with diagonal model in a spectral domain, where it is reasonable to expect that the covariance will be approximately diagonal.

While the spectral diagonal formulation improves the approximation for small ensembles, the spectral diagonal does not converge to the covariance as $N \rightarrow \infty$, unless the covariance is diagonal in the spectral basis.

Equations (9) and (10), respectively Eqs. (12) and (13), can be written in the form

$$
\begin{aligned}
E\left[\left|\mathbf{C}-\mathbf{C}^{N}\right|_{\mathrm{F}}^{2}\right] & =\frac{1}{N-1} \operatorname{Tr}\left(\mathbf{C}^{2}\right)+\frac{1}{N-1}(\operatorname{Tr}(\mathbf{C}))^{2}, \\
E\left[\left|\mathbf{C}-\mathbf{D}^{N}\right|_{\mathrm{F}}^{2}\right] & =\frac{2}{N-1} \operatorname{Tr}\left(\mathbf{C}^{2}\right),
\end{aligned}
$$

using the fact that the trace of a matrix is invariant to similarity transformation. The comparison (Eq. 11) also follows from Eqs. (15) and (16) by noting that $\operatorname{Tr}\left(\mathbf{C}^{2}\right) \leq(\operatorname{Tr}(\mathbf{C}))^{2}$ for all positive semidefinite $\mathbf{C}$, which can be seen, e.g., from Eq. (14).

\section{Spectral EnKF algorithms}

We will show that the analysis step can be implemented very efficiently in cases of practical interest. We drop the ensemble members index in all analysis formulas to make them more readable. Note that when using all the following formulas, it is necessary to perturb the observations.

\subsection{State consisting of only one gridded variable, completely observed}

Assume that the state consists of one gridded variable, e.g., $\boldsymbol{X} \in \mathbb{R}^{n}$, and that we can observe the whole system state, i.e., the observation function is the identity, $\mathbf{H}=\mathbf{I}$, and observations are $\boldsymbol{Y} \in \mathbb{R}^{n}$. Assume also that the observation noise covariance matrix is $c \mathbf{I}$, where $c>0$ is a constant. In this special case we can do the whole update in the spectral space, since it is possible to transform the innovation to the spectral space, and the analysis step (Eq. 8) becomes

$\boldsymbol{X}^{\mathrm{a}}=\boldsymbol{X}-\mathbf{F}^{*} \mathbf{D}_{\mathrm{F}}^{N}\left(\mathbf{D}_{\mathrm{F}}^{N}+c \mathbf{I}\right)^{-1} \mathbf{F}(\boldsymbol{X}-\boldsymbol{Y})$.

Note that the matrices $\mathbf{D}_{\mathbf{F}}^{N}$ and $\mathbf{D}_{\mathrm{F}}^{N}+c \mathbf{I}$ are diagonal, so any operation with them, such as inversion or multiplication, is very cheap. The matrix $\mathbf{F}$ is never formed explicitly. Rather, the multiplications of $\mathbf{F}$ and $\mathbf{F}^{*}$ times a vector are implemented by the FFT or discrete wavelet transform (DWT). This is the base case of both the FFT EnKF (Mandel et al., 2010a, b) and the wavelet EnKF (Beezley et al., 2011).

\subsection{Multiple variables on the same grid, one variable completely observed}

In a typical model, such as numerical weather prediction, the state consists usually of more than one variable. Assume the state consists of $m$ different variables all based on the same grid of length $n$. Then, each variable can be transformed to the spectral space independently, and we have the state vector $X \in \mathbb{R}^{n \cdot m}$ and the transformation matrix in the block form

$\boldsymbol{X}=\left[\begin{array}{c}\boldsymbol{X}_{1} \\ \boldsymbol{X}_{2} \\ \vdots \\ \boldsymbol{X}_{m}\end{array}\right], \quad \mathbf{F}=\left[\begin{array}{cccc}\widetilde{\mathbf{F}} & \mathbf{0} & \cdots & \mathbf{0} \\ \mathbf{0} & \widetilde{\mathbf{F}} & & \vdots \\ \vdots & & \ddots & \mathbf{0} \\ \mathbf{0} & \cdots & \mathbf{0} & \widetilde{\mathbf{F}}\end{array}\right]$,

where each block $\boldsymbol{X}_{1}$ is a vector of length $n$ and $\widetilde{\mathbf{F}}$ is an $n$ by $n$ transformation matrix.

Assume also that the whole state of the first variable $\boldsymbol{X}_{1}$ is observed and, again, that the covariance of observation error is $c \mathbf{I}$. In this case, the observation operator is the one by $m$ block matrix of the form $\mathbf{H}=[\mathbf{I} \mathbf{0} \cdots \mathbf{0}]$. In the proposed method, we approximate the cross-covariances between the variables also by the diagonal of the sample covariance in spectral space, $\mathbf{D}_{\mathrm{F}}^{N}=\left[\mathbf{D}_{i, j}^{N}\right]_{i, j=1}^{m}$, where $\mathbf{D}_{i, j}$ is the matrix containing only diagonal elements from the sample covariance matrix between transformed variables $\widetilde{\mathbf{F}} \boldsymbol{X}_{i}$ and $\widetilde{\mathbf{F}} \boldsymbol{X}_{j}$. With this notation, the analysis step (Eq. 8) becomes

$$
\begin{aligned}
\boldsymbol{X}^{\mathrm{a}}= & {\left[\begin{array}{c}
\boldsymbol{X}_{1}^{\mathrm{a}} \\
\vdots \\
\boldsymbol{X}_{m}^{\mathrm{a}}
\end{array}\right]=\left[\begin{array}{c}
\boldsymbol{X}_{1} \\
\vdots \\
\boldsymbol{X}_{m}
\end{array}\right]-\left[\begin{array}{c}
\widetilde{\mathbf{F}}^{*} \mathbf{D}_{1,1}^{N} \\
\vdots \\
\widetilde{\mathbf{F}}^{*} \mathbf{D}_{m, 1}^{N}
\end{array}\right] } \\
& \left(\mathbf{D}_{1,1}^{N}+c \mathbf{I}\right)^{-1} \widetilde{\mathbf{F}}\left(\boldsymbol{X}_{1}-\boldsymbol{Y}\right) .
\end{aligned}
$$

Note that again the matrix to be inverted is diagonal and full rank, and the transformation $\widetilde{\mathbf{F}}$ is implemented by a call to FFT or DWT, so the operations are computationally very efficient. A related method using interpolation and projection 
was proposed for the case when the model variables are defined on non-matching grids (Beezley et al., 2011).

\subsection{Multiple variables on the same grid, one variable observed at a small number of points}

This situation occurs, e.g., when assimilated observations are from discrete stations. In this case, the observation matrix is $\mathbf{H}=\left[\begin{array}{lll}\mathbf{H}_{1} \mathbf{0} & \cdots & \mathbf{0}\end{array}\right]$, where $\mathbf{H}_{1}$ has a small number of rows, one for each data point, and $\boldsymbol{X}$ and $\mathbf{F}$ are the same as in Eq. (17). We substitute the diagonal spectral approximation into the analysis step (Eq. 8) directly,

$$
\begin{aligned}
\boldsymbol{X}^{\mathrm{a}}= & {\left[\begin{array}{c}
\boldsymbol{X}_{1} \\
\vdots \\
\boldsymbol{X}_{m}
\end{array}\right]-\left[\begin{array}{c}
\widetilde{\mathbf{F}}^{*} \mathbf{D}_{1,1}^{N} \\
\vdots \\
\widetilde{\mathbf{F}}^{*} \mathbf{D}_{m, 1}^{N}
\end{array}\right] \widetilde{\mathbf{F}}\left(\mathbf{H}_{1} \widetilde{\mathbf{F}}^{*} \mathbf{D}_{1,1}^{N} \widetilde{\mathbf{F}} \mathbf{H}_{1}^{*}+\mathbf{R}\right)^{-1} } \\
& \left(\mathbf{H}_{1} \boldsymbol{X}_{1}-\boldsymbol{Y}\right) .
\end{aligned}
$$

The solution of a system of linear equations with the ma$\operatorname{trix} \mathbf{H}_{1} \widetilde{\mathbf{F}}^{*} \mathbf{D}_{1,1}^{N} \widetilde{\mathbf{F}} \mathbf{H}_{1}^{*}+\mathbf{R}$ in Eq. (19) does not present a problem, because its dimension is small by assumption, and $\widetilde{\mathbf{F}} \mathbf{H}_{1}^{*}$ is easy to compute explicitly by the action of FFT on the columns of $\mathbf{H}_{1}^{*}$. Note that in this case, the data noise covariance $\mathbf{R}$ may be arbitrary.

\subsection{State consisting of more variables, one partly observed}

Consider the situation when the number of observation points is too large for the method of Sect. 6.3 to be feasible but only one variable on a part of the mesh is observed. The typical example of this type may be radar images, which cover typically only a part of the domain of the numerical weather prediction model.

The method will go through for any observed subset of entries of the gridded variable $\mathbf{X}_{1}$ but the performance will vary. The performance tends to be better when the observed and unobserved entries of $\mathbf{X}_{1}$ fill two subdomains of the physical domain with a relatively small boundary between them. A detailed investigation, however, is planned for elsewhere.

Suppose that observations $(\boldsymbol{Y})_{j}$ of the values of the first variable $\left(\boldsymbol{X}_{1}\right)_{j}$ are available only for a subset of indices $j \in M \subset\{1, \ldots, n\}$. Augment the forecast state by an additional variable $\boldsymbol{X}_{0}$. For $j=1, \ldots, n$, set $\left(\boldsymbol{X}_{0}\right)_{j}=\left(\boldsymbol{X}_{1}\right)_{j}$ if $j \in M,\left(\boldsymbol{X}_{0}\right)_{j}=(\boldsymbol{Y})_{j}=0$ if $j \notin M$. We can now use the analysis update (Eq. 18) with the augmented state $\widetilde{\boldsymbol{X}}=\left(\boldsymbol{X}_{0}\right.$, $\left.\boldsymbol{X}_{1}, \ldots, \boldsymbol{X}_{m}\right)$ and observation $\tilde{\boldsymbol{Y}}=(\boldsymbol{Y}, \mathbf{0}, \ldots, \mathbf{0})$, to get the augmented analysis $\widetilde{\boldsymbol{X}}^{\mathrm{a}}=\left(\boldsymbol{X}_{0}^{\mathrm{a}}, \boldsymbol{X}_{1}^{\mathrm{a}}, \ldots, \boldsymbol{X}_{m}^{\mathrm{a}}\right)$ and drop $\boldsymbol{X}_{0}^{\mathrm{a}}$.

Note that the innovations to the original variables are propagated through the spectral diagonal approximation of crosscovariance between the original and augmented variables. Since this covariance is not spatially homogeneous, a Fourier basis will not be appropriate, and computational experiments in Sect. 7 confirm that wavelets indeed perform better.

\section{Computational experiments}

In all experiments, we use the usual twin experiment approach. A run of the model from one set of initial conditions is used to generate a sequence of states, which plays the role of the truth. Data values were obtained by applying the observation operator to the truth; the data perturbation was done only for ensemble members within the assimilation algorithm. A second set of initial conditions is used for data assimilation and for a free run, with no data assimilation, for comparison. The error of the free run should be an upper bound on the error of a reasonable data assimilation method.

We evaluate the filter by the root mean square error (RMSE),

$\operatorname{RMSE}=\left(\frac{1}{n} \sum_{i=1}^{n}\left|(\boldsymbol{X})_{i}-(\overline{\boldsymbol{X}})_{i}\right|^{2}\right)^{1 / 2}$,

where $\overline{\boldsymbol{X}}$ is the ensemble mean, forecast or analysis, $\boldsymbol{X}$ is the true state, and $n$ is the number of the grid points $x_{i}$. In the case when the state consists of more than one variable, such as in the shallow water equations, we evaluate the error of each variable independently.

When the true state and the model in the KF evolve following the same mapping (Eq. 1) and the mapping is linear, then the estimate provided by the KF is unbiased and it minimizes the RMSE over all possible gain matrices $\mathbf{K}_{t}$ in Eq. (2). This statistical optimality of the RMSE motivates its use to evaluate how well the data assimilation fulfills its overall purpose to track the truth in the general case when the model is nonlinear and the KF is replaced by the EnKF, with the covariance replaced by an approximation from an ensemble.

We evaluate the RMSE of the standard EnKF, marked as EnKF in the legend of the figures, and the spectral diagonal EnKF with the discrete sine transform, discrete cosine transform, and the Coiflet 2,4 discrete wavelet transform (Daubechies, 1992), marked as DST, DCT, and DWT, respectively.

\subsection{Lorenz 96}

In the Lorenz 96 model (Lorenz, 2006), the state consists of one variable $\boldsymbol{X}_{t} \in \mathbb{R}^{K}, \boldsymbol{X}_{t}=\left(x_{1}, \ldots, x_{K}\right)$, governed by the differential equations

$\frac{\mathrm{d} x_{j}}{\mathrm{~d} t}=x_{j-1} x_{j+1}-x_{j-1} x_{j-2}-x_{j}+F, \quad j=1, \ldots, K$,

where the values of $x_{j-K}$ and $x_{j+K}$ are defined to be equal to $x_{j}$ for each $j=1, \ldots, K$, and $F$ is a parameter.

Our experiments' setup follows the one used in Lorenz and Emanuel (1998). We set the parameter $F=8$, which causes the model to be strongly chaotic. The time step of the model was set to 0.01 time units with assimilation every 0.05 time units, which is equivalent to assimilation into a climatological model every $6 \mathrm{~h}$. The data covariance was diagonal, and 
the standard deviation of observation error was set to $F / 40$. The ensemble and the initial conditions for the truth were generated by sampling from $N\left(F / 4, F^{2} / 4\right)$, and spinup for 18 time units (equivalent to 90 days) was performed. Additionally, while the true state was advanced using the true values of $F=8$, the ensemble members were advanced using the value $0.95 F$ in the Lorenz model.

The only difference from the experiment in Lorenz and Emanuel (1998) was the dimension of the model, where we used 256 instead of 40 . We chose 256 because the dyadic length of the state vector is required when using wavelet transformation, because we wanted to test the proposed augmented algorithm with a significant number of observations and because we wanted to have a significant difference between ensemble size and state dimension. To test the chaotic properties of this model we performed two independent simulations with very close initial conditions and measured the difference in each time step between the states using maximum norm. Initial values for the first simulation were generated as i.i.d. random variables from $N\left(F / 4, F^{2} / 4\right)$, and the initial values for the second simulation were created by perturbing the first set of initial values with white noise with variance of 0.0001 . We performed this experiment for both state dimensions, 40 and 256. The results (Fig. 1a) show that the change of the state dimension does not affect the rate of divergence of two initially close solutions. Figure $1 \mathrm{~b}$ and c show one solution of the Lorenz 96 model with state dimensions 40 and 256, respectively, after 50 time units for illustration of the chaotic character of the state.

In the case when the whole state is observed, spectral filters with ensemble size $N=4$ (Fig. 2a) already decrease the error significantly compared to a run with no assimilation, while the standard EnKF actually increases the error. For all filters, the error eventually decreases with the ensemble size at the standard rate $N^{-1 / 2}$; however, the spectral EnKF shows the error decrease from the start, while the EnKF lags until the ensemble size is comparable to the state dimension and even then its RMSE is significantly higher (Fig. 2b).

Next, consider the case when only the first $m$ points of a grid are observed. In the legend, DCT-S and DWT-S are the methods with the discrete cosine transform and the Coiflet 2,4 discrete wavelet transform, respectively, with the standard analysis update (Eq. 8), while DCT-A and DWTA use the augmented state methods from Sect. 6.4. Figure 3 shows that the spectral diagonal method decreases the RMSE, while the standard EnKF is unstable. This observation is consistent with the result of Kelly et al. (2014), which shows that, for a class of dynamical systems, the EnKF remains within a bounded distance of truth if sufficiently large covariance inflation is used and if the whole state is observed. The augmented state method DWT-A with wavelet transformation gave almost the same analysis error as DCT-S, which is using the spectral diagonal filter with the exact observation matrix, while the cosine basis, which implies a homogeneous random field, resulted in a much larger error (method DCT-
A). A similar behavior was seen with a smaller number of observed points as well, but the error reduction in the spectral diagonal EnKF was smaller (not shown).

\subsection{Shallow water equations}

The shallow water equations can serve as a simplified model of atmospheric flow. The state $\boldsymbol{Y}=(h, u, v)$ consists of water level height $h$ and velocities $u$ and $v$ in the $x$ and $y$ directions, governed by the differential equations of conservation of mass and momentum:

$$
\begin{aligned}
& \frac{\partial h}{\partial t}+\frac{\partial(u h)}{\partial x}+\frac{\partial(v h)}{\partial y}=0, \\
& \frac{\partial(h u)}{\partial t}+\frac{\partial}{\partial x}\left(h u^{2}+\frac{1}{2} g h^{2}\right)+\frac{\partial(h u v)}{\partial y}=0, \\
& \frac{\partial(h v)}{\partial t}+\frac{\partial(h u v)}{\partial x}+\frac{\partial}{\partial y}\left(h v^{2}+\frac{1}{2} g h^{2}\right)=0,
\end{aligned}
$$

where $g$ is gravity acceleration, with reflective boundary conditions and without Coriolis force or viscosity. The equations were discretized on a rectangular grid size of $64 \times 64$ with a horizontal distance between grid points of $150 \mathrm{~km}$ and advanced by the Lax-Wendroff method with the time step $1 \mathrm{~s}$. The initial values were water level $h=10 \mathrm{~km}$, plus Gaussian drop with height of $1 \mathrm{~km}$, width of 32 nodes, in the center of the domain, and $u=v=0$. See Moler (2011, Chapter 18) for details.

We have used two independent initial conditions, one used for the truth and another for the ensemble and the free run. The only difference was the location of the initial wave. Both states were moved forward for $3 \mathrm{~h}$. Then the ensemble was created by adding random noise (with prescribed background covariance). Then, all states were moved forward for another $3 \mathrm{~h}$ and assimilation starts $6 \mathrm{~h}$ after the model initialization. All assimilation methods start with the same forecast in the first assimilation cycle. The 2-D tensor products FFT and DWT were used in the diagonal spectral EnKF. The observation error was assumed to have zero mean and variance of $1000 \mathrm{~m}^{2}$ in $h$ and $1000 \mathrm{~kg} \mathrm{~m} \mathrm{~s}^{-1}$ in $u$ and $v$.

The background covariance for initial ensemble perturbation was estimated using samples taken every minute, from time $t_{\text {start }}=3 \mathrm{~h}$ to time $t_{\mathrm{end}}=6 \mathrm{~h}$, and modified by tapering the sample covariance matrix $\mathbf{C}_{N}$ as $\mathbf{B}=\mathbf{C}_{N} \circ \mathbf{T}$, where the tapering matrix $\mathbf{T}$ had the block structure

$\mathbf{T}=\left[\begin{array}{ccc}\mathbf{A} & \mathbf{0} & \mathbf{0} \\ \mathbf{0} & \mathbf{A} & \mathbf{0} \\ \mathbf{0} & \mathbf{0} & \mathbf{A}\end{array}\right]+0.9\left[\begin{array}{ccc}\mathbf{0} & \mathbf{A} & \mathbf{A} \\ \mathbf{A} & \mathbf{0} & \mathbf{A} \\ \mathbf{A} & \mathbf{A} & \mathbf{0}\end{array}\right]$,

where the entry between nodes $\left(i_{a}, j_{a}\right)$ and $\left(i_{b}, j_{b}\right)$ is (A) $)_{a, b}=\exp \left(-\left|i_{a}-i_{b}\right|\right) \exp \left(-\left|j_{a}-j_{b}\right|\right)$. Note that matrix $\mathbf{T}$ could be also rewritten using the Kronecker product as $\mathbf{T}=\mathbf{K} \otimes(\mathbf{M} \otimes \mathbf{M})$, where $\mathbf{K}$ is a $3 \times 3$ square matrix with elements $(\mathbf{K})_{i, i}=1,(\mathbf{K})_{i, j}=0.9$ if $i \neq j$ and $\mathbf{M}$ is a $64 \times 64$ square matrix with elements $(\mathbf{M})_{i, j}=\exp (|i-j|)$. Since 

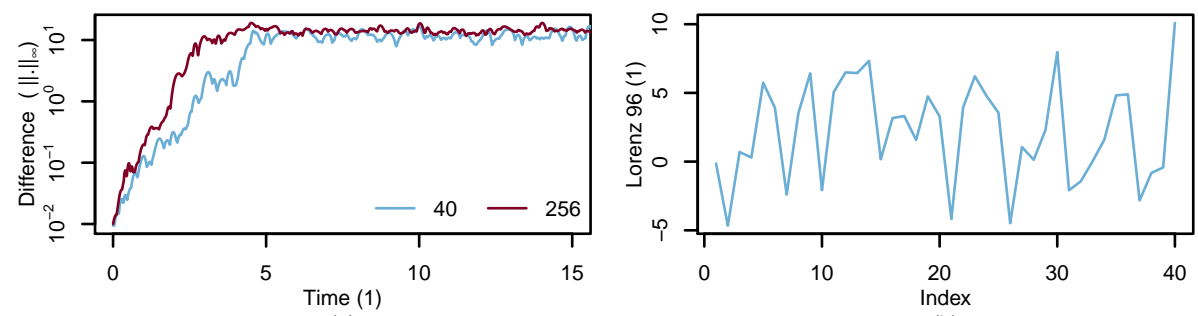

(a)

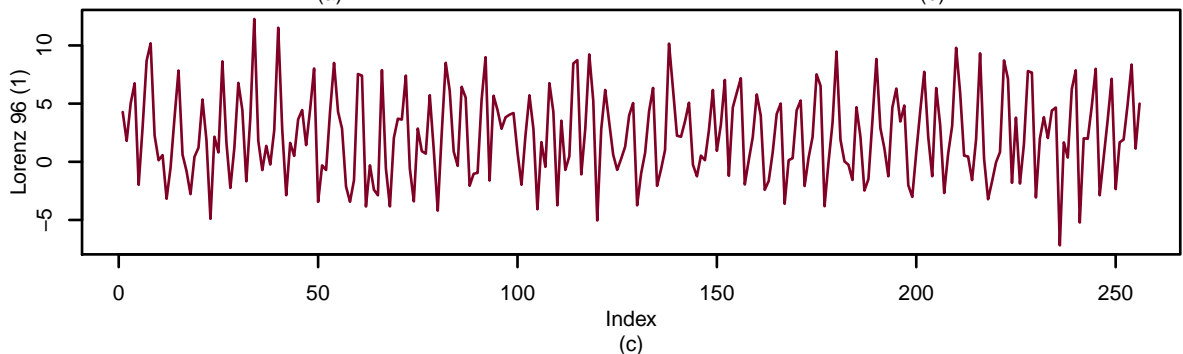

Figure 1. (a) Growth of the difference in the maximum norm $|\cdot|_{\infty}$ of two initially close solutions of the Lorenz 96 model. The initial states differ by white noise with variance of $10^{-4}$. The growth of the difference is shown for the state dimensions of 40 and for the state dimension 256. (b) A solution of the Lorenz 96 model with state dimension 40 after 50 time units. (c) A solution of the Lorenz 96 model with state dimension 256 after 50 time units.

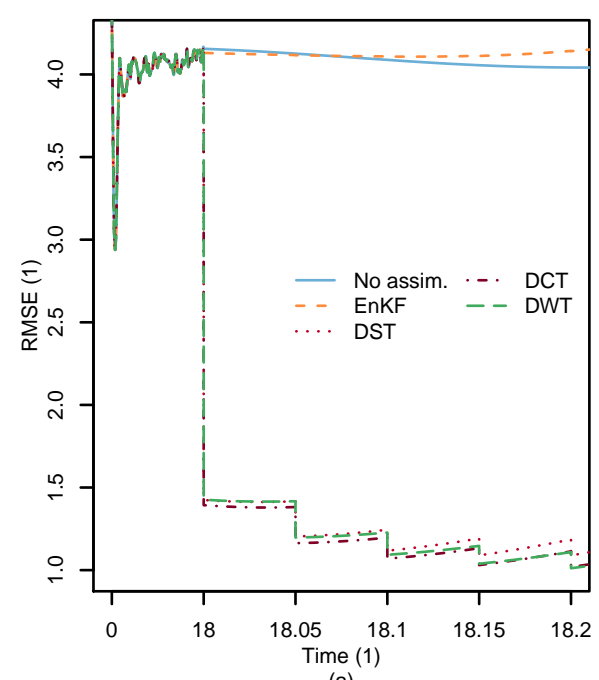

(a)

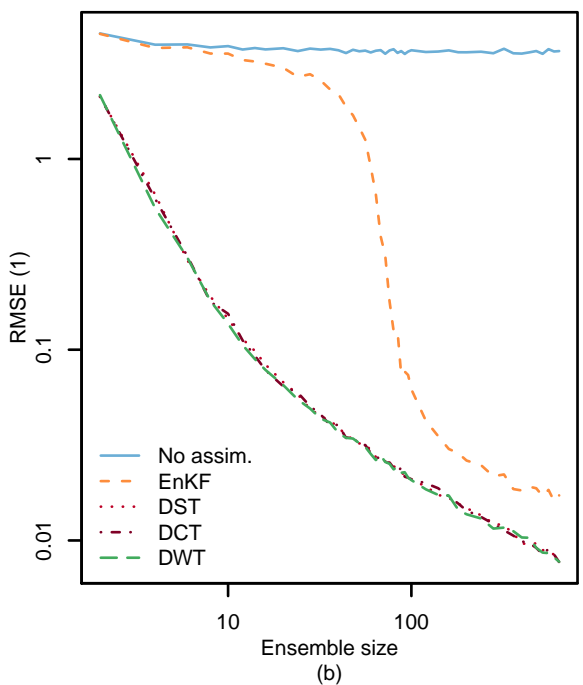

Figure 2. Mean RMSE from 10 realizations for the Lorenz 96 problem, the whole state observed (a) increasing analysis cycles with ensemble size 4, state dimension 256 and (b) increasing ensemble size, analysis cycle 1, state dimension 64.

both matrices $\mathbf{K}$ and $\mathbf{M}$ are positive definite, matrix $\mathbf{T}$ is also positive definite.

When the full state is observed, the spectral diagonal method decreased the RMSE in all variables dramatically (Fig. 4), unlike the standard EnKF. When only the water level is observed, the RMSE in spectral diagonal EnKF decreases less but is still much more than that in the standard EnKF (Fig. 5).

\section{Conclusions}

A version of the ensemble Kalman filter was presented, based on replacing the sample covariance by its diagonal in the spectral space, which provides a simple, efficient, and automatic localization. We have demonstrated efficient implementations for several classes of observation operators and data important in applications, including high-dimensional data defined on a part of the domain, such as radar or satellite images. The spectral diagonal was proved rigorously to give a lower mean square error than the sample covariance. Computational experiments with the Lorenz 96 problem and the 

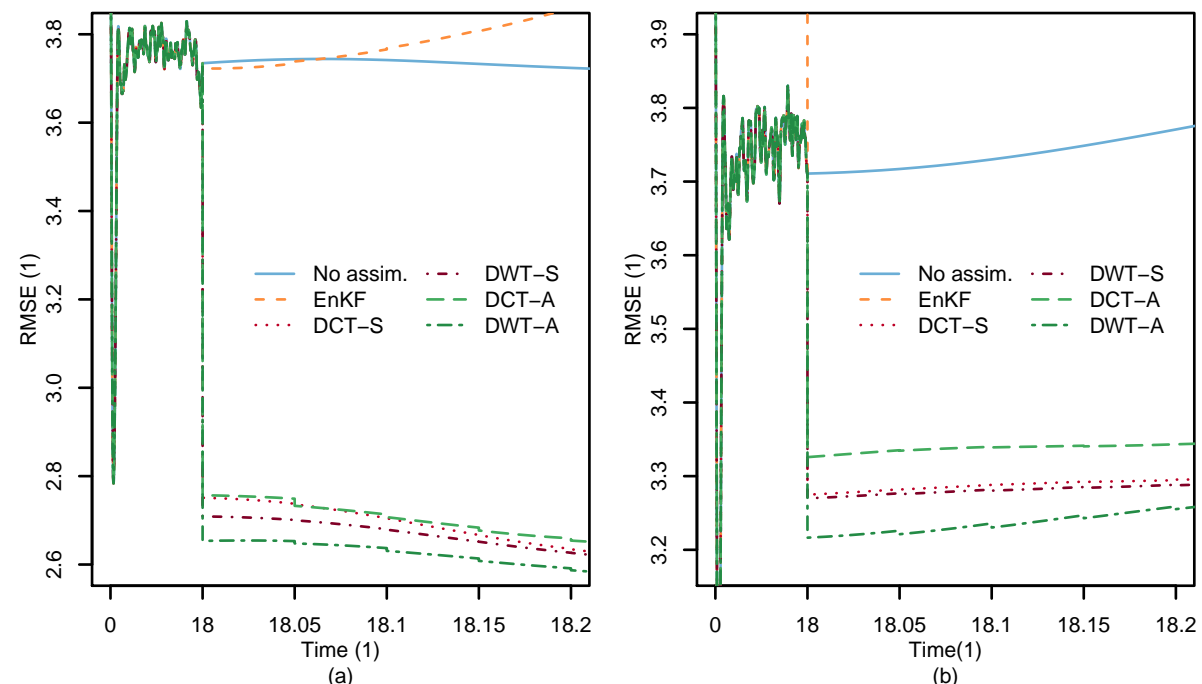

Figure 3. Mean RMSE from 10 realizations for the Lorenz 96 problem, ensemble size 16, state dimension 256. (a) First 128 points observed and (b) first 64 points observed.

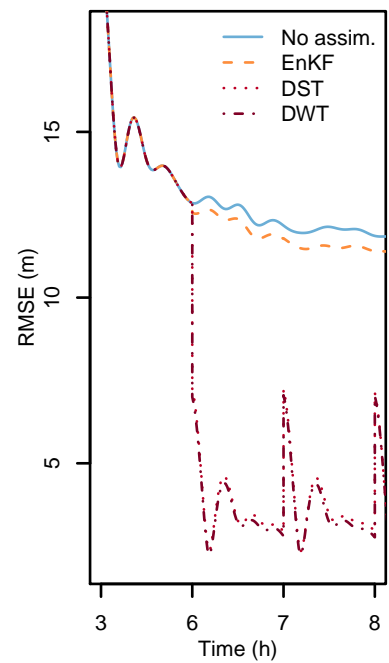

(a)

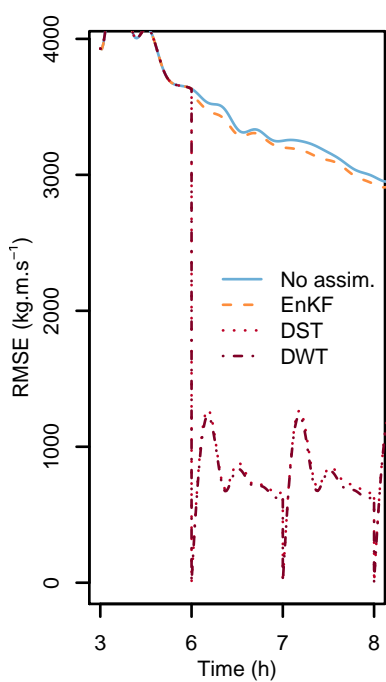

(b)

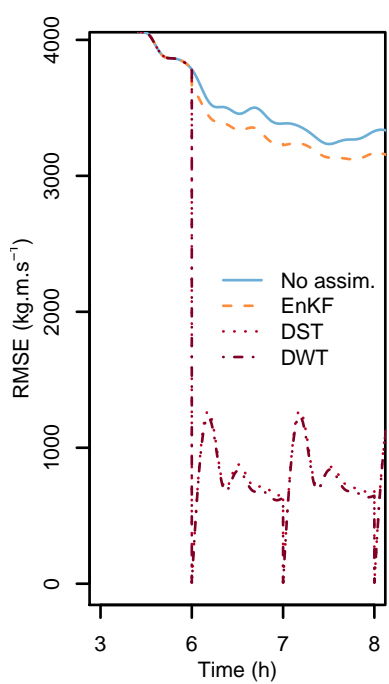

(c)

Figure 4. RMSE of ensemble mean of one realization of three assimilation cycles. Full state was observed. The length of assimilation cycle is $60 \mathrm{~min}$, ensemble size 20. (a) Water height, (b) velocity in the $x$ direction and (c) velocity in the $y$ direction.

shallow water equations have shown that the analysis error drops very fast for small ensembles, and the method is stable over multiple analysis cycles. The paper provides a technique for data assimilation which can work with minimal computational resources because an implementation needs only an orthogonal transformation, such as the fast Fourier or discrete wavelet transform, and manipulation of vectors and diagonal matrices. Therefore, it should be of interest in applications.

The present method uses orthogonal transformation, but orthogonality is not a necessary condition for a diagonal assumption in general; diagonal approximation with frames was proposed in Pannekoucke et al. (2007). The question of further reducing the number of parameters and thus sampling noise as in, e.g., functions of the Laplace operator, is also of interest. When a different spectral diagonalization is used for each horizontal plane, the question is how to connect horizontal sheets along the vertical dimension. In Pannekoucke (2008, their Appendix D), wavelet packets are used to take advantage of the orthogonal basis dictionary they provide. These issues will be studied elsewhere.

The method described in Sect. 6.2 is general and it allows for arbitrary linear observation operators, but an inverse (i.e., solving a system) in the observation space is required. The computational cost then grows as the cube of data dimension. This issue is well known in spectral variational methods; techniques used in the literature include aggregat- 

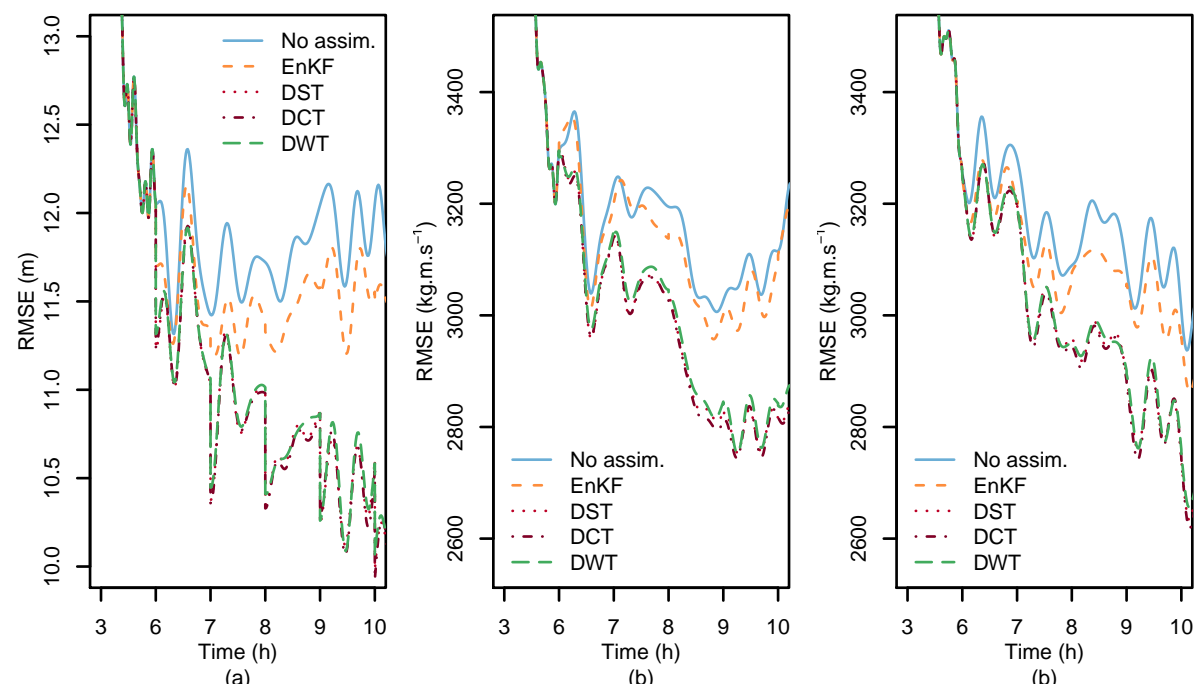

Figure 5. Mean RMSE of ensemble mean from five independent repetitions. Ensemble size 20, only water height observed. (a) Water height, (b) velocity in the $x$ direction and (c) velocity in the $y$ direction.

ing and interpolating observations to create "super observations" as gridded arrays (Parrish and Derber, 1992). 
Appendix A: Error estimate of sample covariance matrix

We prove an extension of Mallat (1998, Prop. 10.14) to sample covariance of a random vector with unknown mean.

\section{A1 Lemma 1}

Let $\boldsymbol{U}^{k} \sim N(\boldsymbol{\mu}, \mathbf{C}), k=1, \ldots, N$, be i.i.d. vectors in $\mathbb{R}^{n}$ or $\mathbb{C}^{n}$, and

$$
\begin{aligned}
\left(\mathbf{C}^{N}\right)_{i, j}= & \frac{1}{N-1}\left(\sum_{k=1}^{N}\left(\left(\boldsymbol{U}^{k}\right)_{i}-\frac{1}{N} \sum_{l=1}^{N}\left(\boldsymbol{U}^{k}\right)_{i}\right)\right. \\
& \left.\left(\left(\boldsymbol{U}^{k}\right)_{j}-\frac{1}{N} \sum_{l=1}^{N}\left(\boldsymbol{U}^{l}\right)_{j}\right)^{*}\right)
\end{aligned}
$$

their sample covariance. Then,

$$
E\left[\left|\left(\mathbf{C}^{N}\right)_{i, j}-(\mathbf{C})_{i, j}\right|^{2}\right]=\frac{1}{N-1}\left(\left|(\mathbf{C})_{i, j}\right|^{2}+(\mathbf{C})_{i, i}(\mathbf{C})_{j, j}\right) .
$$

\section{A2 Proof}

The proof follows that of Mallat (1998, Prop. 10.14) with adjustments for the presence of the sample mean in Eq. (A1). Each element of the sample covariance $c_{i, j}^{N}=\left(\mathbf{C}^{N}\right)_{i, j}$ is an unbiased estimate of the covariance $c_{i, j}=(\mathbf{C})_{i, j}$, so

$E\left[\left|c_{i, j}^{N}-c_{i, j}\right|^{2}\right]=E\left[\left|c_{i, j}^{N}\right|^{2}\right]-\left|c_{i, j}\right|^{2}$.

Without loss of generality, assume $\boldsymbol{\mu}=\mathbf{0}$, subtracting the constant $\boldsymbol{\mu}$ if necessary, and compute

$$
\begin{aligned}
E\left[\left|c_{i, j}^{N}\right|^{2}\right] & =E[ \\
& \ldots \sum_{m=1}^{N}\left(u_{j}^{m}\right)^{* 2} \frac{1}{(N-1)^{2}} E\left[\left|\sum_{k=1}^{N} u_{i}^{k}\left(u_{j}^{k}\right)^{*}\right|^{2}\right] \\
& -\frac{1}{N(N-1)^{2}} E\left[\sum_{k, l, m=1}^{N} u_{i}^{k}\left(u_{j}^{k}\right)^{*}\left(u_{i}^{l}\right)^{*} u_{j}^{m}\right] \\
& -\frac{1}{N(N-1)^{2}} E\left[\sum_{k, l, m=1}^{N}\left(u_{i}^{k}\right)^{*} u_{j}^{k} u_{i}^{l}\left(u_{j}^{m}\right)^{*}\right] \\
& +\frac{1}{N^{2}(N-1)^{2}} \mathrm{E}\left[\left|\sum_{l, m=1}^{N} u_{i}^{l}\left(u_{j}^{m}\right)^{*}\right|^{2}\right] .
\end{aligned}
$$

Now we utilize the Isserlis theorem, also known as Wick's formula, which states that if $A_{1}, A_{2}, A_{3}$, and $A_{4}$ have a jointcentered Gaussian distribution, then

$$
\begin{aligned}
E\left[A_{1} A_{2} A_{3} A_{4}\right] & =E\left[A_{1} A_{2}\right] E\left[A_{3} A_{4}\right]+E\left[A_{1} A_{3}\right] E\left[A_{2} A_{4}\right] \\
& +E\left[A_{1} A_{4}\right] E\left[A_{2} A_{3}\right]
\end{aligned}
$$

cf. Isserlis (1918). Since our samples are independent and $E\left[u_{i}^{k}\right]=0$, we know that

$E\left[u_{i}^{k}\left(u_{j}^{k}\right)^{*}\right]=c_{i j}, \quad E\left[u_{i}^{k} u_{j}^{l}\right]=0$ if $k \neq l$,

and we get

$$
\begin{aligned}
E\left[u_{i}^{k}\left(u_{j}^{l}\right)^{*}\left(u_{i}^{m}\right)^{*} u_{j}^{n}\right] & \left.=\left|c_{i, j}\right|^{2} \mathbf{1}_{\{k=l, m=n\}}+c_{i, i} c_{j, j} \mathbf{1}_{\{k=m, l=n\}}\right\} \\
& +E\left[u_{i} u_{j}\right] E\left[\left(u_{j}\right)^{*}\left(u_{i}\right)^{*}\right] \mathbf{1}_{\{k=n, l=m\}} .
\end{aligned}
$$

Applying this equation in Eq. (A2), we get

$$
E\left[\left|c_{i, j}^{N}\right|^{2}\right]=\frac{1}{N-1}\left(c_{i, i} c_{j, j}+N\left|c_{i, j}\right|^{2}\right),
$$

and the final result follows

$$
\begin{aligned}
E\left[\left|c_{i, j}^{N}-c_{i, j}\right|^{2}\right] & =\frac{1}{N-1}\left(c_{i, i} c_{j, j}+N\left|c_{i, j}\right|^{2}\right)-\left|c_{i, j}\right|^{2} \\
& =\frac{1}{N-1}\left(c_{i, i} c_{j, j}+\left|c_{i, j}\right|^{2}\right) .
\end{aligned}
$$


Acknowledgements. The authors would like to thank the anonymous reviewers and the editor, whose comments have contributed to improving the paper. This research was partially supported by the Czech Science Foundation under the grant GA13-34856S and the US National Science Foundation under the grant DMS-1216481. A part of this research was done when Ivan Kasanický and Martin Vejmelka visited the University of Colorado Denver.

Edited by: O. Talagrand

Reviewed by: two anonymous referees

\section{References}

Anderson, B. D. O. and Moore, J. B.: Optimal Filtering, PrenticeHall, Englewood Cliffs, NJ, 1979.

Anderson, J. L.: An ensemble adjustment Kalman filter for data assimilation, Mon. Weather Rev., 129, 2884-2903, doi:10.1175/1520-0493(2001)129<2884:AEAKFF>2.0.CO;2, 2001.

Beezley, J. D., Mandel, J., and Cobb, L.: Wavelet ensemble Kalman filters, in: Vol. 2, Proceedings of IEEE IDAACS'2011, 15-17 September 2011, Prague, 514-518, doi:10.1109/IDAACS.2011.6072819, 2011.

Berre, L.: Estimation of synoptic and mesoscale forecast error covariances in a limited-area model, Mon. Weather Rev., 128, 644-667, doi:10.1175/15200493(2000)128<0644:EOSAMF>2.0.CO;2, 2000.

Berre, L., Pannekoucke, O., Desroziers, G., Stefanescu, S., Chapnik, B., and Raynaud, L.: A variational assimilation ensemble and the spatial filtering of its error covariances: increase of sample size by local spatial averaging, http://old.ecmwf.int/publications/library/ecpublications/ _pdf/workshop/2007/Data_assimilation/Berre.pdf (last access 7 August 2015), 2007.

Boer, G. J.: Homogeneous and isotropic turbulence on the sphere, J. Atmos. Sci., 40, 154-163, doi:10.1175/15200469(1983)040<0154:HAITOT>2.0.CO;2, 1983.

Buehner, M.: Ensemble-derived stationary and flow-dependent background-error covariances: Evaluation in a quasi-operational NWP setting, Q. J. Roy. Meteorol. Soc., 131, 1013-1043, doi:10.1256/qj.04.15, 2005.

Buehner, M. and Charron, M.: Spectral and spatial localization of background-error correlations for data assimilation, Q. J. Roy. Meteorol. Soc., 133, 615-630, doi:10.1002/qj.50, 2007.

Burgers, G., van Leeuwen, P. J., and Evensen, G.: Analysis scheme in the ensemble Kalman filter, Mon. Weather Rev., 126, 17191724, 1998.

Courtier, P., Andersson, E., Heckley, W., Vasiljevic, D., Hamrud, M., Hollingsworth, A., Rabier, F., Fisher, M., and Pailleux, J.: The ECMWF implementation of three-dimensional variational assimilation (3D-Var), I: Formulation, Q. J. Roy. Meteorol. Soc., 124, 1783-1807, doi:10.1002/qj.49712455002, 1998.

Da Prato, G.: An Introduction to Infinite-Dimensional Analysis, Springer-Verlag, Berlin, doi:10.1007/3-540-29021-4, 2006.

Daubechies, I.: Ten Lectures on Wavelets, Vol. 61, CBMS-NSF Regional Conference Series in Applied Mathematics, Society for
Industrial and Applied Mathematics (SIAM), Philadelphia, PA, doi:10.1137/1.9781611970104, 1992.

Deckmyn, A. and Berre, L.: A Wavelet Approach to Representing Background Error Covariances in a Limited-Area Model, Mon. Weather Rev., 133, 1279-1294, doi:10.1175/MWR2929.1, 2005.

Derber, J. and Bouttier, F.: A reformulation of the background error covariance in the ECMWF global data assimilation system, Tellus A, 51, 195-221, doi:10.1034/j.1600-0870.1999.t012-00003.x, 1999.

Evensen, G.: Data Assimilation: The Ensemble Kalman Filter, 2nd Edn., Springer, Berlin, doi:10.1007/978-3-642-03711-5, 2009.

Fisher, M.: Background error covariance modelling, in: Proceedings of the ECMWF Seminar on Recent Developments in Data Assimilation for Atmosphere and Ocean, ECMWF, Reading, UK, 45-63, 2003.

Fisher, M. and Andersson, E.: Developments in 4D-Var and Kalman Filtering, ECMWF Technical Memorandum 347, available at http://www.ecmwf.int/publications/library/ecpublications/_pdf/ tm/301-400/tm347.pdf (last access: November 2013), 2001.

Furrer, R. and Bengtsson, T.: Estimation of high-dimensional prior and posterior covariance matrices in Kalman filter variants, J. Multivariate Anal., 98, 227-255, doi:10.1016/j.jmva.2006.08.003, 2007.

Gaspari, G. and Cohn, S. E.: Construction of correlation functions in two and three dimensions, Q. J. Roy. Meteorol. Soc., 125, 723757, doi:10.1002/qj.49712555417, 1999.

Hamill, T. M. and Snyder, C.: A Hybrid Ensemble Kalman Filter-3D Variational Analysis Scheme, Mon. Weather Rev., 128, 2905-2919, doi:10.1175/15200493(2000)128<2905:AHEKFV>2.0.CO;2, 2000.

Hunt, B. R., Kostelich, E. J., and Szunyogh, I.: Efficient data assimilation for spatiotemporal chaos: a local ensemble transform Kalman filter, Physica D, 230, 112-126, doi:10.1016/j.physd.2006.11.008, 2007.

Isserlis, L.: On a Formula for the Product-Moment Coefficient of any Order of a Normal Frequency Distribution in any Number of Variables, Biometrika, 12, 134-139, 1918.

Kalman, R. E.: A new approach to linear filtering and prediction problems, J. Basic Eng.-T. ASME, 82, 35-45, doi:10.1115/1.3662552, 1960.

Kalnay, E.: Atmospheric Modeling, Data Assimilation and Predictability, Cambridge University Press, 2003.

Kelly, D. T. B., Law, K. J. H., and Stuart, A. M.: Well-posedness and accuracy of the ensemble Kalman filter in discrete and continuous time, Nonlinearity, 27, 2579-2603, doi:10.1088/09517715/27/10/2579, 2014.

Kepert, J. D.: Covariance localisation and balance in an Ensemble Kalman Filter, Q. J. Roy. Meteorol. Soc., 135, 1157-1176, doi:10.1002/qj.443, 2009.

Kwiatkowski, E. and Mandel, J.: Convergence of the square root ensemble Kalman filter in the large ensemble limit, SIAM/ASA J. Uncert. Quantificat., 3, 1-17, doi:10.1137/140965363, 2015.

Lahoz, W., Khattatov, B., and Menard, R. (Eds.): Data Assimilation: Making Sense of Observations, Springer, Berlin, doi:10.1007/978-3-540-74703-1, 2010.

Le Gland, F., Monbet, V., and Tran, V.-D.: Large sample asymptotics for the ensemble Kalman filter, in: The Oxford Handbook 
of Nonlinear Filtering, edited by: Crisan, D. and Rozovskii, B., Oxford University Press, 598-631, 2011.

Lorenz, E. N.: Predictability - a problem partly solved, in: Predictability of Weather and Climate, edited by: Palmer, T. and Hagendorn, R., Cambridge University Press, 40-58, 2006.

Lorenz, E. N. and Emanuel, K. A.: Optimal Sites for Supplementary Weather Observations: Simulation with a Small Model, J. Atmos. Sci., 55, 399-414, doi:10.1175/15200469(1998)055<0399:OSFSWO>2.0.CO;2, 1998.

Mallat, S.: A wavelet tour of signal processing, Academic Press, Inc., San Diego, CA, 1998.

Mandel, J., Beezley, J. D., Coen, J. L., and Kim, M.: Data Assimilation for Wildland Fires: Ensemble Kalman filters in coupled atmosphere-surface models, IEEE Control Syst. Mag., 29, 4765, doi:10.1109/MCS.2009.932224, 2009.

Mandel, J., Beezley, J., Cobb, L., and Krishnamurthy, A.: Data driven computing by the morphing fast Fourier transform ensemble Kalman filter in epidemic spread simulations, Proced. Comput. Sci., 1, 1215-1223, doi:10.1016/j.procs.2010.04.136, 2010a.

Mandel, J., Beezley, J. D., and Kondratenko, V. Y.: Fast Fourier transform ensemble Kalman filter with application to a coupled atmosphere-wildland fire model, in: Computational Intelligence in Business and Economics, Proceedings of MS'10, Barcelona, edited by: Gil-Lafuente, A. M. and Merigo, J. M., World Scientific, 777-784, doi:10.1142/9789814324441_0089, 2010b.

Mandel, J., Cobb, L., and Beezley, J. D.: On the convergence of the ensemble Kalman filter, Appl. Math.-Czech, 56, 533-541, doi:10.1007/s10492-011-0031-2, 2011.

Ménétrier, B., Montmerle, T., Michel, Y., and Berre, L.: Linear Filtering of Sample Covariances for Ensemble-Based Data Assimilation, Part I: Optimality Criteria and Application to Variance Filtering and Covariance Localization, Mon. Weather Rev., 43, 1622-1643, doi:10.1175/MWR-D-14-00157.1, 2015.

Michel, Y.: Estimating deformations of random processes for correlation modelling in a limited area model, Q. J. Roy. Meteorol. Soc., 139, 534-547, doi:10.1002/qj.1978, 2013.

Moler, C.: Experiments with MATLAB, available at: http://www. mathworks.com/moler/exm (last access: December 2014), 2011.

Pannekoucke, O., Berre, L., and Desroziers, G.: Filtering properties of wavelets for local background-error correlations, Q. J. Roy. Meteorol. Soc., 133, 363-379, doi:10.1002/qj.33, 2007.

Pannekoucke, O.: Modélisation des structures locales de covariance des erreurs de prévision à l'aide des ondelettes, $\mathrm{PhD}$ thesis, Université Paul Sabatier-Toulouse III, Toulouse, https://tel. archives-ouvertes.fr/tel-00285515/document (last access: 7 August 2015), 2008.
Pannekoucke, O.: Heterogeneous Correlation Modeling Based on the Wavelet Diagonal Assumption and on the Diffusion Operator, Mon. Weather Rev., 137, 2995-3012, doi:10.1175/2009MWR2783.1, 2009.

Pannekoucke, O. and Massart, S.: Estimation of the local diffusion tensor and normalization for heterogeneous correlation modelling using a diffusion equation, Q. J. Roy. Meteorol. Soc., 134, 1425-1438, doi:10.1002/qj.288, 2008.

Pannekoucke, O., Emili, E., and Thual, O.: Modelling of local length-scale dynamics and isotropizing deformations, Q. J. Roy. Meteorol. Soc., 140, 1387-1398, doi:10.1002/qj.2204, 2014.

Parrish, D. F. and Derber, J. C.: The National Meteorological Center's Spectral Statistical-Interpolation analysis system, Mon. Weather Rev., 120, 1747-1763, doi:10.1175/15200493(1992)120<1747:TNMCSS>2.0.CO;2, 1992.

Purser, R. J., Wu, W.-S., Parrish, D. F., and Roberts, N. M.: Numerical Aspects of the Application of Recursive Filters to Variational Statistical Analysis, Part II: Spatially Inhomogeneous and Anisotropic General Covariances, Mon. Weather Rev., 131, 1536-1548, doi:10.1175//2543.1, 2003.

Raynaud, L. and Pannekoucke, O.: Sampling properties and spatial filtering of ensemble background-error length-scales, Q. J. Roy. Meteorol. Soc., 139, 784-794, doi:10.1002/qj.1999, 2013.

Raynaud, L., Berre, L., and Desroziers, G.: Objective filtering of ensemble-based background-error variances, Q. J. Roy. Meteorol. Soc., 135, 1177-1199, doi:10.1002/qj.438, 2009.

Sakov, P. and Bertino, L.: Relation between two common localisation methods for the EnKF, Computat. Geosci., 15, 225-237, doi:10.1007/s10596-010-9202-6, 2011.

Varella, H., Berre, L., and Desroziers, G.: Diagnostic and impact studies of a wavelet formulation of background-error correlations in a global model, Q. J. Roy. Meteorol. Soc., 137, 1369-1379, doi:10.1002/qj.845, 2011.

Weaver, A. and Courtier, P.: Correlation modelling on the sphere using a generalized diffusion equation, Q. J. Roy. Meteorol. Soc., 127, 1815-1846, doi:10.1002/qj.49712757518, 2001.

Weaver, A. T., Deltel, C., Machu, E., Ricci, S., and Daget, N.: A multivariate balance operator for variational ocean data assimilation, Q. J. Roy. Meteorol. Soc., 131, 3605-3625, doi:10.1256/qj.05.119, 2005. 